\title{
Fabrication of Y-junction Metal Nanowires by AAO Template-assisted AC Electrodeposition
}

\author{
Huanan Duan', Zhenhai Xia² and Jianyu Liang1,*
}

In this communication, we report a synthetic approach to fabricate Y-junction Co nanowires and Y-junction $\mathrm{Cu}$ nanowires by AC electrodeposition using a hierarchically designed anodized aluminum oxide template. Morphology study showe that diameters of the stems and branches of the Y-junction nanowires were about $40 \mathrm{~nm}$ and $20 \mathrm{~nm}$ respectively. Structural analysis indicates that Co nanowires had a mixture of face-center-cubic and hexagonal-close-packed structures, whereas $\mathrm{Cu}$ nanowires had a face-center-cubic structure with a $<110>$ texture. The Y-junction Co nanowires exhibited a longitudinal coercivity of 1300 Oe and remnant magnetization of 56\%, which was affected by the growth direction and microstructure. The present method can be extended to other metallic systems and thus provides a simple and efficient way to fabricate Y-junction metal nanowires.

Keywords: AAO template; Nanofabrication; AC electrodeposition; Y-junction metal nanowires; Magnetic property

Citation: Huanan Duan, Zhenhai Xia and Jianyu Liang, "Fabrication of Y-junction Metal Nanowires by AAO Template-assisted AC Electrodeposition”, Nano-Micro Lett. 2, 290-295 (2010). doi:10.3786/nml.v2i4.p290-295

Metal nanowires (NWs) have attracted vigorous research interests in recent years [1-11]. Among the numerous synthesis methods studied so far, fabrication inside rationally designed anodic aluminum oxide (AAO) templates has been proved to be an economic and versatile method to produce nanostructures with great efficiency and precision [2-4]. The AAO templates have many desirable characteristics such as a narrowly distributed pore size which can be tuned within a wide range, a well-developed fabrication process, easiness to remove, good mechanical and thermal stability, and chemical inertness. Since the pioneer work by T. M. Whitney [5], AAO template-assisted fabrication has achieved great success in the synthesis of linear metal nanowires [2-4,6-11]. Particularly, in applications such as nanoelectronics where nanowires with more complicated structure, such as Y-junction or branched NWs, are desirable, AAO template-assisted fabrication offers a simple and efficient method for the preparation of Y-junction metal NWs. Although in the past few years, a few reports were available on the synthesis of Y-junction carbon nanotubes $[12,13]$ and metallic
Y-junction or branched nanowires by direct current (DC) electrodeposition [14-15], limited progress has been made on the synthesis of metallic Y-junction or branched nanowires by alternate current (AC) electrodeposition [20]. A typical synthesis process by DC electrodeposition is as following: firstly, the AAO templates are separated from the $\mathrm{Al}$ substrate; after the removal of the barrier layer, the templates are coated with a thin layer of noble metal to make an electrode; then target metal is deposited into the templates by $\mathrm{DC}$ electrodeposition. While $\mathrm{AC}$ electrodeposition has been proven to be a simple method to fabricate linear metal nanowires [6-11], using AC electrodeposition to fabricate metallic Y-junction NWs certainly demands more systematic exploration. Moreover, how the crystal structure and magnetic property of the synthesized Y-junction NWs are affected by the AC electrodeposition remains unclear.

In this paper, we report a generic synthetic approach to fabricate Y-junction metallic NWs including $\mathrm{Co}$ NWs and $\mathrm{Cu}$ NWs by AC electrodeposition using AAO templates with 
well-controlled Y-junction channels. The morphology and structure of the NWs were characterized by scanning electron microscopy (SEM), transmission electron microscopy (TEM), selected area electron diffraction (SAED), and X-ray diffraction (XRD). The magnetic property of the Y-junction Co NWs was investigated by a conventional vibrating-sample magnetometer (VSM).

A schematic of the synthesis steps is shown in Fig. 1. The AAO templates were obtained by a well-established two-step anodization process [21-23]. Briefly, the first anodic oxidation of aluminum $(99.999 \%$ pure, Electronic Space Products International) was carried out in a $0.3 \mathrm{M}$ oxalic acid solution at $40 \mathrm{~V}$ and $10^{\circ} \mathrm{C}$ for $16-20 \mathrm{~h}$. The porous alumina layer formed during this first anodization step was completely dissolved by a mixture solution of $6 \%$ phosphoric acid and $1.8 \%$ chromic acid at $70^{\circ} \mathrm{C}$. The sample was then subjected to a second anodization where initially the anodization was performed under the same conditions as in the first anodization to produce the primary stem pores, and then the anodizing voltage was reduced by a factor of $1 / \sqrt{2}$ to create the Y-branched pores. The as-prepared AAO templates were wet etched in $0.5 \% \mathrm{H}_{3} \mathrm{PO}_{4}$ for half an hour to thin the barrier layer and widen the pores.

Cobalt nanowires were electrochemically deposited by AC electrolysis in this nanoporous template with $\mathrm{Y}$-junction nanochannels using $14 \mathrm{Vrms}$ at $100 \mathrm{~Hz}$ for $30 \mathrm{~min}$ in an electrolyte solution consisting of $240 \mathrm{~g} \cdot \mathrm{l}^{-1}$ of $\mathrm{CoSO}_{4} \cdot 7 \mathrm{H}_{2} \mathrm{O}$ (Alfa Aesar), $40 \mathrm{~g} \cdot l^{-1}$ of $\mathrm{HBO}_{3}$ (Alfa Aesar), and $1 \mathrm{~g} \cdot \mathrm{l}^{-1}$ of ascorbic acid (Alfa Aesar) [21-23]. Graphite was used as the counter electrode.

The synthesis of copper nanowires was adopted from G. A. Gelves's work $[10,11]$. Electrodeposition of the Y-junction $\mathrm{Cu}$ NWs was carried out in an aqueous solution consisting of $0.50 \mathrm{M}$ $\mathrm{CuSO}_{4}$ (Alfa Aesar) and $0.285 \mathrm{M} \mathrm{H}_{3} \mathrm{BO}_{3}$ by applying a continuous $200 \mathrm{~Hz}$ sine wave at $10 \mathrm{Vrms}$ for $10 \mathrm{~min}$ between the anodized $\mathrm{Al}$ and the graphite counter electrode.

XRD patterns were recorded on a Rigaku Miniflex diffractometer using a $\mathrm{Cu} \mathrm{K} \alpha \mathrm{X}$-ray source (1.5405 $\AA$ ). The structure and morphology were characterized by SEM using a JEOL JSM-7000F microscope and by TEM using a Phillips CM 12 operated at an accelerating voltage of $120 \mathrm{kV}$. TEM samples were prepared as follows: first the $\mathrm{Y}$-junction $\mathrm{NWs}$ were liberated from the AAO template by dissolving the template in 2 $\mathrm{M} \mathrm{NaOH}$ for 1 hour. After rinsing with DI water and dispersing the NWs in ethonal by sonication, a few drops of the solution were placed onto a carbon-coated $\mathrm{Cu}$ grid and dried in air. The magnetic property of the as-prepared Y-junction Co NWs was investigated using VSM with magnetic field in the range of $-10,000$ Oe to 10,000 Oe at room temperature.

Dimensions of the Y-junction AAO channels and the Y-junction metal NWs were analyzed using the image processing software of Image J [24]. Dimensions were measured at ten different locations on each of multiple SEM and TEM images and the average and standard deviation were reported.

The top-view SEM image (Fig. 2a) shows that nanopores within the AAO template form a highly ordered hexagonal pattern. The cross-section view SEM image (Fig. 2b) exhibits 5 micrometer long well-defined Y-junction channels within the AAO template. An arbitrary green line has been drawn to indicate the interface between the branches and the stems. Examination of Fig. $2 b$ with Image $J$ reveals that diameters of the stems and the branches of the Y-junction channels are $39.2 \pm 4.2$ $\mathrm{nm}$ and $19.6 \pm 4.4 \mathrm{~nm}$, respectively. It is notable that while the diameters of the branches are usually half that of the stems when the branches are formed at $1 / \sqrt{ } 2$ of the voltage for the formation of the stems, the lengths of the stems and the branches of the

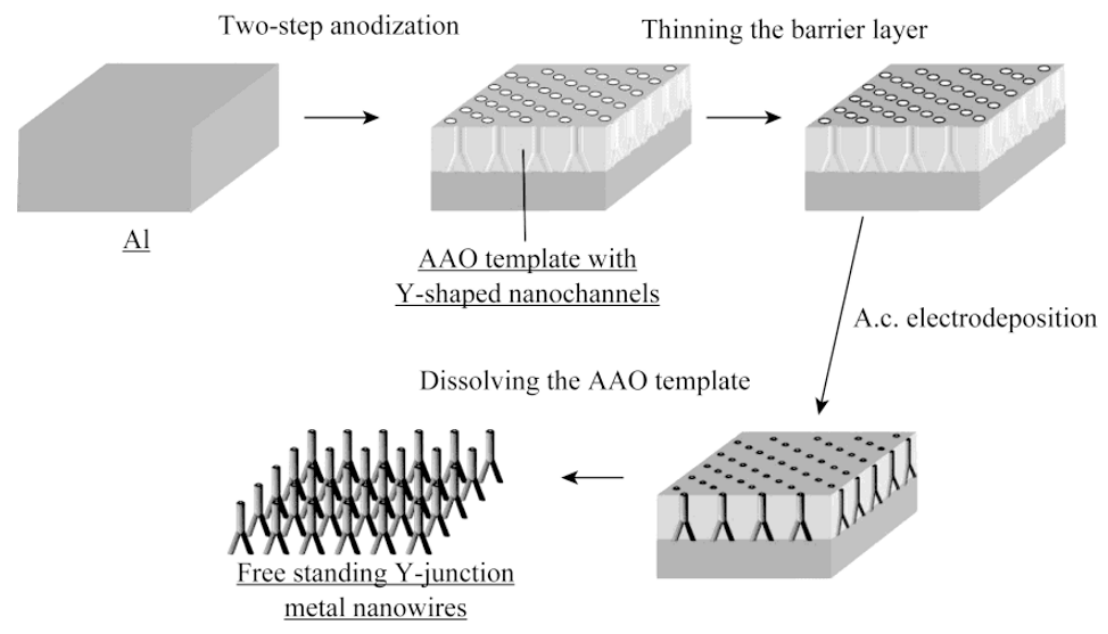

FIG. 1. Schematic of the process to synthesize Y-junction metal NWs. 

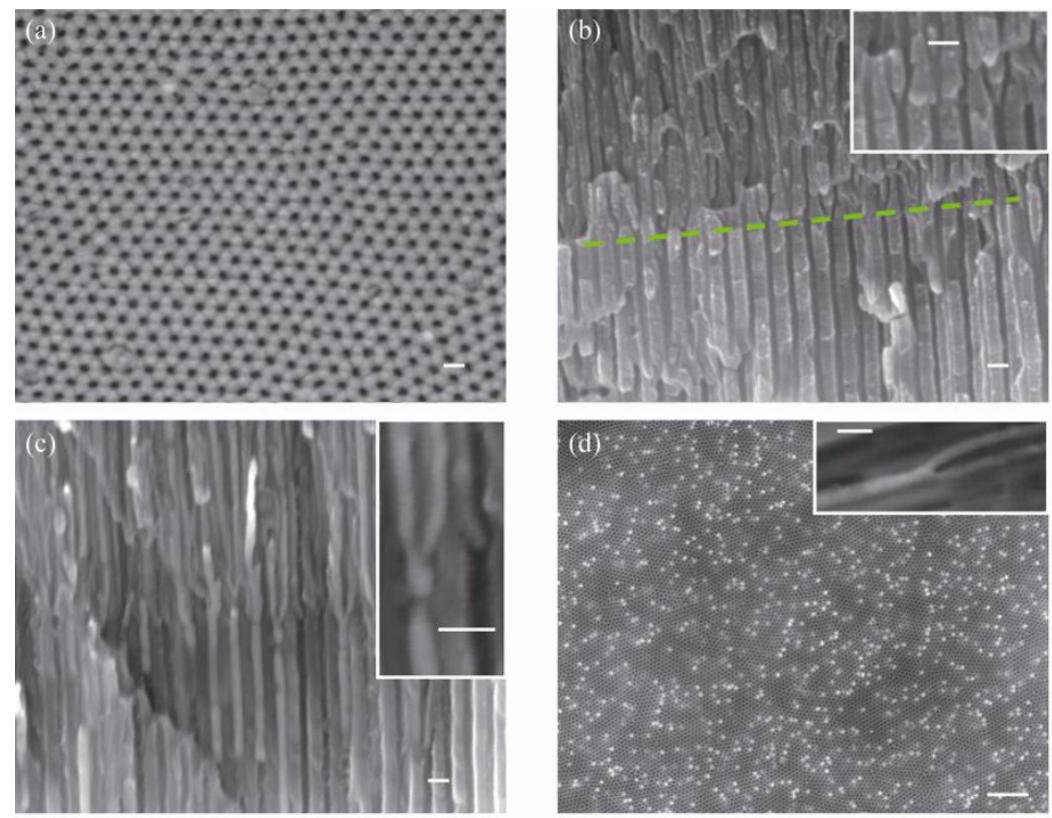

FIG. 2. SEM images of AAO templates with Y-junction nanochannels (a), (b), and after electrodeposition of Co (c) and Cu (d). Scale bar: $100 \mathrm{~nm}$ in all figures except the inset of (d); $1 \mu \mathrm{m}$ in the inset of (d).

Y-junction channels can be independently adjusted by controlling the anodization duration for each segment.

Typical SEM images of the Y-junction Co NWs and Y-junction $\mathrm{Cu}$ NWs are shown in Fig. 2c and d. Clearly, NWs are parallel to each other and well contained in the Y-branched nanochannels. Statistical measurement by Image J shows that for Y-junction Co NWs, diameters of the stems and the branches are $39.3 \pm 5.6 \mathrm{~nm}$ and $20.9 \pm 4.8 \mathrm{~nm}$, respectively; while for Y-junction $\mathrm{Cu}$ NWs, diameters of the stems and the branches are $40.3 \pm 4.6 \mathrm{~nm}$ and $21.9 \pm 4.2 \mathrm{~nm}$, respectively. These diameters are in close agreement with the dimension of the Y-junction AAO nanochannels. Figure $2 \mathrm{~d}$ also shows that 10 min deposition of $\mathrm{Cu}$ NWs can fill up some of 5 micrometer long AAO nanochannels.

XRD pattern for the Y-junction Co NWs embedded in the AAO template (Fig. 3(a)) shows that other than the Al peaks associated with the Al substrate (PDF card no. 04-0787), all peaks correspond to Co. Moreover, the Co NWs consist of a mixture of face-center-cubic (FCC) (PDF card no. 15-0806) and hexagonal-close-packed (HCP) (PDF card no. 05-0727) structures. The peak near $76^{\circ}$ could be a combination of the diffraction from the (110) plane of the HCP structure and the (220) plane of the FCC structure, and the peak near $92.5^{\circ}$ could be a combination of the diffraction from the (112) plane of the HCP structure and the (311) plane of the FCC structure. The coexistence of FCC and HCP structures has been observed in the electrodeposited straight Co NWs in previous studies using DC, $\mathrm{AC}$, or pulsed deposition techniques [25-28]. It suggests a complex growth mechanism because HCP and FCC Co NWs are generally believed to be obtained by two distinct mechanisms, namely, two-dimensional layer-by-layer growth and three-
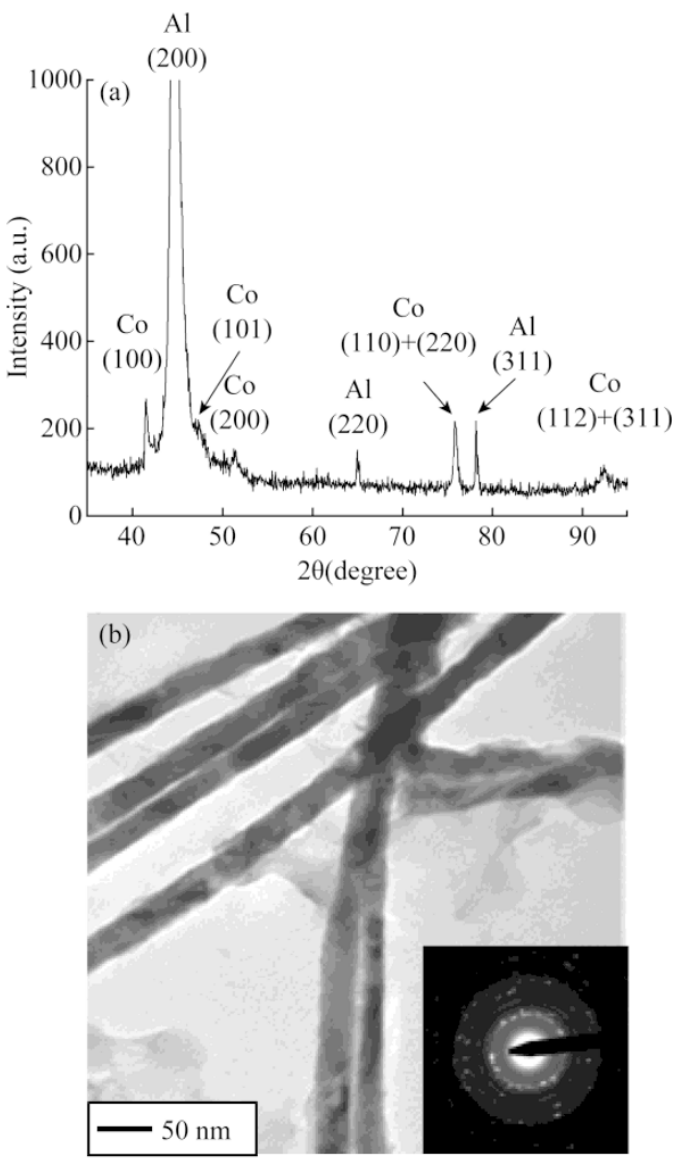

FIG. 3. (a) XRD pattern for the Y-junction Co NWs embedded in AAO template; (b) TEM of the Y-junction Co NWs. Inset: SAED patterns for (b). 
dimensional nucleation/growth [29, 30]. The growth mechanism can be affected by the synthesis conditions such as $\mathrm{pH}$ value of the electrolyte [29], frequency of the power source [31], and the deposition potential [32].

Figure $3 \mathrm{~b}$ shows a bright-field TEM image of Y-junction Co NWs. SAED was performed to investigate the crystalline structures of the Y-junction Co NWs. The broken ring SAED patterns suggest that the structures are polycrystalline in nature. The patterns are complicated due to the change of growth direction at the junction as well as the coexistence of HCP and FCC structures as shown in XRD results.

Due to the reduced dimension and the Y-shape, Y-junction Co NWs may possess unique magnetic properties. VSM was used to investigate the magnetic properties of the as-synthesized Y-junction Co NWs embedded in the AAO template. The hysteresis loops were recorded with the applied magnetic field parallel and perpendicular to the nanowire axis, respectively, at room temperature. As shown in Fig. 4, the coercivity, Hc, measured with the magnetic field perpendicular (transverse) to the wire axis is determined to be $486 \mathrm{Oe}$, much higher than $15 \mathrm{Oe}$, the value of straight single-crystal Co NWs reported [36]. In contrast, when the magnetic field is parallel (longitudinal) to the wire axis, $H_{C}$ is determined to be $1300 \mathrm{Oe}$, around three times greater than the transverse value. The longitudinal $H_{C}$ for straight Co NWs was reported in the range of 1100 2000 Oe [35, 36]. This enhanced longitudinal $H_{C}$ suggests that the wire axis was the preferred overall magnetization direction. However, this enhancement was much less compared to straight Co NWs. Figure 4 also shows that the longitudinal remnant magnetization $\left(M_{r}\right)$ equals to $56 \%$ of the saturated magnetization $\left(M_{S}\right)$. The remnant magnetization is lower compared to that of the straight Co NWs, which was in the range of $67.8-80 \%$ [34-36]. We attribute this difference in the shape anisotropy and remnant

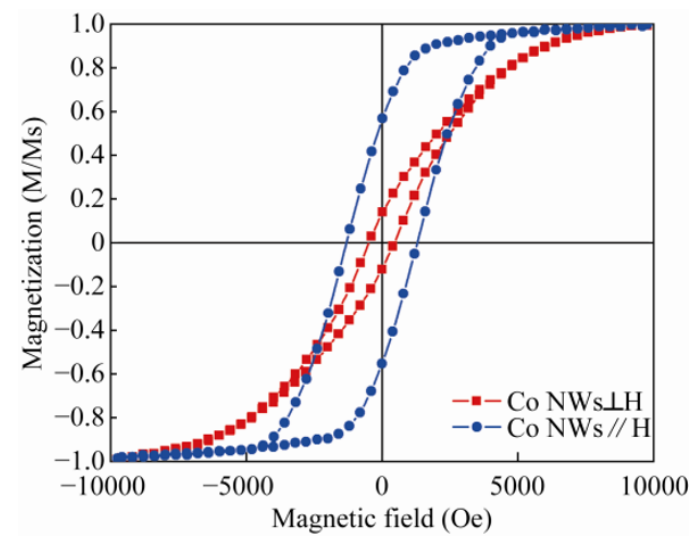

FIG. 4. Hysteresis behavior of the Y-junction Co nanowires with magnetic field $H$ parallel and perpendicular to wire axis. magnetization to the unique structure of the as-synthesized Y-junction Co NWs. For Y-junction Co NWs, the splitting of Co NWs and the change of growth direction near the $\mathrm{Y}$ junction affect the shape anisotropy. Moreover, the coexistence of HCP and FCC crystal structures by AC electrodeposition is another important factor because the coexistence may incur more complexity, inhomogeneity, and more domain structures compared to single-crystal Co NWs. It has been reported that for straight Co NWs with same diameters, the effective anisotropy along the nanowire axis of the FCC Co NW array is stronger than that of the HCP Co NW array [25].

The XRD pattern for the Y-junction $\mathrm{Cu}$ NWs (Fig. 5(a)) shows that the $\mathrm{Cu}$ NWs have a FCC structure (PDF card number: 04-0836) exhibiting a $<110>$ texture, which is interesting because for bulk FCC structures the most energetically favorable texture is $<111>$. One of the possible reason is that the adsorption of hydrogen may result in the stabilization of the (110) face during the electrodeposition of NWs in acidic solutions [33]. Moreover, at high overpotential the electrodeposited $\mathrm{Cu}$ NWs may undergo a thermodynamic to kinetic transition, producing [110] orientation [34].

Figure $5 \mathrm{~b}$ depicts the TEM images of Y-junction $\mathrm{Cu}$ NWs. Analysis by Image $\mathrm{J}$ shows that the stems and branches of
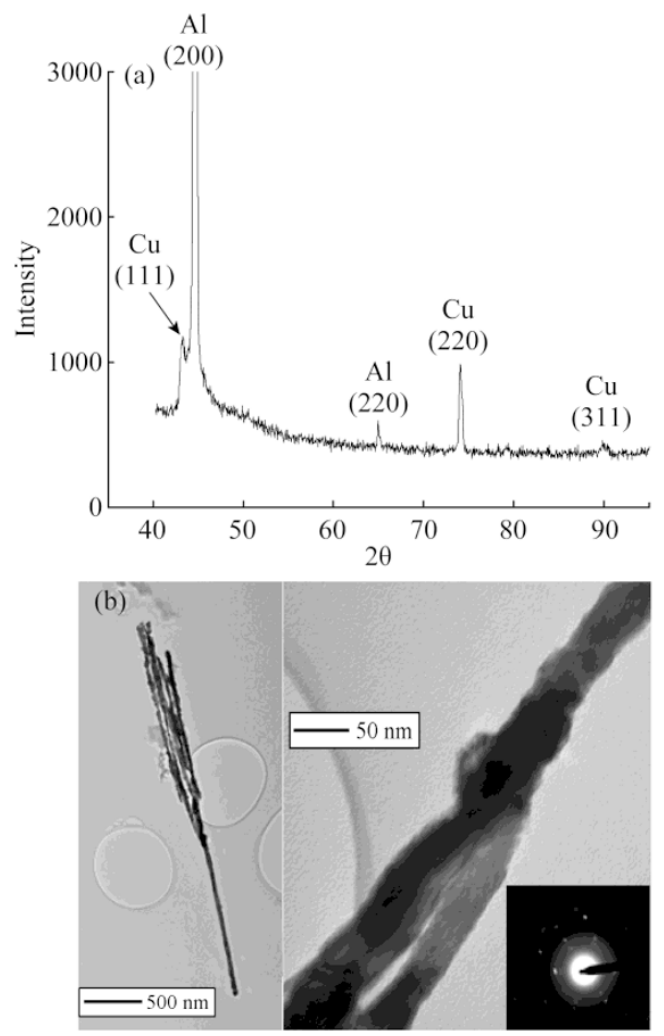

FIG. 5. (a) XRD pattern for the Y-junction $\mathrm{Cu}$ NWs embedded in AAO template; (b) TEM of the Y-junction Cu NWs. Inset: SAED patterns for (b) taken along the [111] zone axis perpendicular to the long axis of the NWs. 
individual Y-junction $\mathrm{Cu}$ NWs have diameters of $42.8 \pm 7.4 \mathrm{~nm}$ and $35.8 \pm 5.3 \mathrm{~nm}$, respectively. Similar analysis of TEM images of Y-junction Co NWs shows that diameters of the stems and branches are $42.2 \mathrm{~nm} \pm 4.0 \mathrm{~nm}$ and $28.1 \mathrm{~nm} \pm 4.2 \mathrm{~nm}$, respectively. Clearly, the diameters of the stems and the branches of both metal NWs are considerably larger compared with the results estimated by SEM images. The dimensional increase may be due to the surface oxidation during the template removal or TEM sample preparation process [37]. The spotty diffraction rings in Fig. 5(b) (inset) show the polycrystalline nature of the $\mathrm{Cu}$ NWs.

In summary, by using a hierarchically designed AAO template, we fabricated Y-junction Co NWs and Y-junction $\mathrm{Cu}$ NWs by AC electrodeposition. Morphology study showed that well-defined Y-junctions were synthesized and dimensions of the NWs were defined by the template. Structure analysis indicated that the Co NWs were a mixture of FCC and HCP structures, and $\mathrm{Cu}$ NWs had FCC structure with a $<110>$ texture. The coexistence of the FCC and HCP structures affected the coercivity and magnetization squareness of the Co NWs. The present method can be extended to other metallic systems and thus provide a simple and efficient way to fabricate Y-junction metal NWs.

This work is financially supported partially by a NSF award CMMI-0825990.

\section{Received 7 November 2010; accepted 7 January 2011; published} online 20 January 2011.

\section{References}

1. J. Wang, L. Y. Zhang, P. Liu, T. M. Lan, J. Zhang, L. M. Wei, E. S. Kong, C. H. Jiang and Y. F. Zhang, Nano-Micro Lett. 2, 134-138 (2010). doi:10.5101/nml. v2i2.p134-138

2. H. Masuda, F. Hasegwa and S. Ono, J. Electrochem. Soc. 144, L127 (1997). doi:10.1149/1.1837634

3. A. P. Li, F. Muller, A. Birner, K. Nielsch and U. Gosele, J. Appl. Phys. 84, 6023 (1998). doi:10.1063/1.368911

4. O. Jessensky, F. Muller and U. Gosele, Appl. Phys. Lett. 72, 1173 (1998). doi:10.1063/1.121004

5. T. M. Whitney, J. S. Jiang, P. C. Searson and C. L. Chien, Science 261, 1316 (1993). doi:10.1126/science. $\underline{261.5126 .1316}$

6. A. J. Yin, J. Li, W. Jian, A. J. Bennett and J. M. Xu, Appl. Phys. Lett. 79, 1039 (2001). doi:10.1063/1.1389765
7. X. Y. Zhang, G. H. Wen, Y. F. Chan, R. K. Zheng, X. X. Zhang and N. Wang, Appl. Phys. Lett. 83, 3341 (2003). doi:10.1063/1.1621459

8. N. J. Gerein and J. A. Haber, J. Phys. Chem. B 109, 17372 (2005). doi:10.1021/jp051320d

9. R. L. Wang, S. L. Tang, B. Nie, X. L. Fei, Y. G. Shi and Y. W. Du, Solid State Comm. 142, 639 (2007). doi:10.1016/j.ssc.2007.04.014

10. G. A. Gelves, B. Lin, U. Sundararaj and J. A. Haber, Adv. Funct. Mater. 16, 2423 (2006). doi:10.1002/ adfm.200600336

11. G. A. Gelves, Z. T. Murakami, M. M. J. Krantz and J. A. Haber, J. Mater. Chem. 16, 3075 (2006). doi:10.1039/ $\underline{b 603442 j}$

12. J. Li, C. Papadopoulos and J. Xu, Nature 402, 253 (1999). doi: $10.1038 / 46214$

13. G. Meng, Y. J. Jung, A. Cao, R. Vajtai and P. M. Ajayan, Proc. Natl. Acad. Sci. 102, 7074 (2005). doi:10.1073/ pnas.0502098102

14. T. Gao, G. Meng, J. Zhang, S. Sun and L. Zhang, Appl. Phys. A: Mater. Sci. Process. 74, 403 (2002). doi:10.1007/s003390101136

15. J. Choi, G. Sauer, K. Nielsch, R. B. Wehrspohn and U. Gosele, Chem. Mater. 15, 776 (2003). doi:10.1007/ s003390101136

16. Y. Tian, G. Meng, S. K. Biswas, P. M. Ajayan, S. Sun and L. Zhang, Appl. Phys. Lett. 85, 967 (2004). doi:10.1063/ 1.1779956

17. S. Mahima, R. Kannan, I. Komath, M. Aslam and V.K. Pillai, Chem. Mater. 20, 601 (2008). doi:10.1021/ $\mathrm{cm} 702102 \mathrm{~b}$

18. D. Li, C. Jiang, J. Jiang and J. G. Lu, Chem. Mater. 21, doi:10.1021/cm8022242

19. B. Chen, Q. Xu, X. Zhao, X. Zhu, M. Kong and G. Meng, Adv. Funct. Mater. 20, 3791 (2010). doi:10.1002/ adfm. 201001190

20. J. Zhang, C. S. Day and D. L. Carroll, Chem. Comm. 45, doi:10.1039/b913917f

21. J. Liang, H. Chik, A. Yin and J. M. Xu, J. Appl. Phys. 91, 2544 (2002). doi:10.1063/1.1433173

22. J. Liang, H. Chik and J. M. Xu, IEEE J. Sel. Top. Quantum Electron. 8, 998 (2002). doi:10.1109/ JSTQE.2002.804238

23. J. Li, C. Papadopoulos, J. M. Xu and M. Moskovits, Appl. Phys. Lett. 75, 367 (1999). doi:10.1063/1.124377 
24. http://rsbweb.nih.gov/ij/

25. F. Li, T. Wang, L. Ren and J. Sun, J. Phys.: Condens. Matter. 16, 8053 (2004). doi:10.1088/0953-8984/ $\underline{16 / 45 / 027}$

26. G. Tourillon, L. Pontonnier, J. P. Levy and V. Langlais, Electrochem. Solid-State Lett. 3, 20 (2000). doi:10.1149/ $\underline{1.1390946}$

27. G. J. Strijkers, J. H. J. Dalderop, M. A. A. Broeksteeg, H. J. M. Swagten and W. J. M. de Jonge, J. Appl. Phys. 86, 5141 (1999). doi:10.1063/1.371490

28. N. R. Pradhan, H. Duan, J. Liang and G. S. Iannacchione, $\begin{array}{llll}\text { Nanotech. } & 19, \quad \text { (2008). doi:10.1088/ }\end{array}$ $\underline{0957-4484 / 19 / 48 / 485712}$

29. H. Pan, H. Sun, C. Poh, Y. Feng and J. Lin, Nanotech. 16, 1559 (2005). doi:10.1088/0957- 4484/16/9/025

30. M. Tian, J. Wang, J. Kurtz, T. E. Mallouk and M. H. Chan, Nano Lett. 3, 919 (2003). doi:10.1021/n1034217d

31. J. Zhang, G. A. Jones, T. H. Shen, S. E. Donnely and G. Li,
J. Appl. Phys. 101, 054310 (2007). doi:10.1063/ $\underline{1.2464193}$

32. X. W. Wang, G. T. Fei, P. Tong, X. J. Xu and L. D. Zhang, J. Crystal Growth 300, 421 (2007). doi:10.1016/ j.jcrysgro.2006.12.039

33. J. A. Switzer, H. M. Kothari and E. W. Bohannan, J. Phys. Chem. B 106, 4027 (2002). doi:10.1021/jp014638o

34. H. Pan, B. Liu, J. Yi, C. Poh, S. Lim, J. Ding, Y. Feng, C. H. A. Huan and J. Lin, J. Phys. Chem. B 109, 3094 (2005). doi:10.1021/jp0451997

35. M. Darques, A. Encinas, L. Vila and L. Piraux, J. Phys. D: Appl. Phys. 37, 1411 (2004). doi:10.1088/0022-3727/ $\underline{37 / 10 / 001}$

36. Z. Liu, P. C. Chang, C. C. Chang, E. Galaktionov, G. Bergmann and J. G. Lu, Adv. Funct. Mater. 18, 1573 (2008). doi:10.1002/adfm.200701010

37. N. J. Gerein and J. A. Haber, J. Phys. Chem. B 109, 17372 (2005). doi:10.1021/jp051320d 